\title{
Application of Carbon Dioxide and Polyacrylamide in Extracting Magnesium from Brine by Precipitation Method
}

\author{
Haiyan Hu, Lin Zhou, Qingguo Chen \\ Marine Science and Technology College, Zhejiang Ocean University, Zhoushan, China \\ Email: huhaiyan@zjou.edu.cn
}

Received 3 June 2016; accepted 27 July 2016; published 30 July 2016

\begin{abstract}
In this paper, carbon dioxide and polyacrylamide were used to improve the method to abstract magnesium from desalination brine. Experimental results showed that calcium ion in the desalination brine can be effectively removed using carbon dioxide, which thereby improves the purity and reaction efficiency of the magnesium hydrate precipitate. $0.1 \%-0.2 \%$ PAM could help to promote the precipitation of magnesium hydrate colloid. And when hydroxyl anion was between $110 \%-130 \%$ of theoretical amount, yield of magnesium hydrate exceeds $70 \%$ at standing time of 60 hours.
\end{abstract}

\section{Keywords}

\section{Magnesium Element, Desalination Brine, Reverse Osmosis}

\section{Introduction}

Magnesium is an important industrial raw material, which can not only be used as a reductant to recover metals like titanium, zirconium, uranium and beryllium, but also can be used to make fireworks, flash powder, fireproof materials, etc. With structural properties similar to aluminum, magnesium can be used as an alloy material for aircrafts and missiles. In addition to the industrial sectors, magnesium is also used in medical, sports, chemical reagent manufacturing and other areas.

Magnesium is a widely-distributed element, which was obtained mainly by ore smelting in the past. In recent years, with the acceleration of the marine resources development, seawater has also become an important source of magnesium. Magnesium content in seawater and brine ranks only next to sodium and chlorine, and thus is worth exploring. Meanwhile, with the rapid development of seawater desalination industry, extraction of magnesium from desalination brine has also gained researchers' attention. Since most of elements contained in desalination brine are higher in concentration than those in normal seawater, obtaining equivalent amounts of chemical resources from desalination brine is easier than extracting from normal seawater. In addition, chemical resource extraction from desalination brine can greatly save investment and engineering costs because it does not 
require additional pretreatment equipment such as seawater intake, chlorination facilities, and so on.

At present, magnesium is extracted from brine mainly by precipitation method [1]. Alkali is added into the brine to produce magnesium hydroxide precipitate to separate magnesium from the brine through phase separation, which is the most rapid and effective method [2]. In this process, two core problems need to be addressed: one is the interference by calcium ions; and the other is the improvement of magnesium ion precipitation efficiency. In this study, the extents to which these two difficulties can be resolved are investigated through experiments using two chemicals, carbon dioxide $\left(\mathrm{CO}_{2}\right)$ and polyacrylamide (PAM).

\section{Materials and Methods}

\subsection{Experimental Materials}

(1) $\mathrm{CO}_{2}(99 \%)$; polyacrylamide (A.R.)

(2) Desalination brine was collected from the brine outfall for desalination project of Liuheng Water Services Ltd. in Zhoushan City, Zhejiang Province, China.

\subsection{Experimental Methods}

\subsubsection{Removal of Calcium}

Preliminary experiments have shown that magnesium hydrate obtained by precipitation had poor purity when desalination brine was directly used as the raw material, with a calcium content of about $3 \%-7 \%$. If a solution removed of calcium was operated previously, calcium content would be reduced to less than $1 \%$. In an alkaline environment, carbon dioxide was allowed to preferentially react with calcium ion in the desalination brine, then the prepared decalcified mother liquor containing low calcium ion was reacted with alkali to produce the desired product magnesium hydrate. In this way, the purity of magnesium precipitate could be improved [3]-[5].

In the first set of experiments, $200.00 \mathrm{~mL}$ of brine was placed in a three-necked flat-bottom flask; speed of magnetic stirrer was controlled at 600 rpm; $p H$ meter was inserted below the liquid level; and $p H$ value was recorded after the reading stabilized. Next, carbon dioxide (flow rate of about $0.5 \mathrm{~L} \cdot \mathrm{min}^{-1}$ ) was passed through the solution; meanwhile, $2.5 \mathrm{~mol} \cdot \mathrm{L}^{-1}$ sodium hydroxide solution was added dropwise. Timing started when the $p H$ of the system changed to $8 \pm 0.2$. After reacting for certain times ( $1 \mathrm{~min}, 2 \mathrm{~min}, 3 \mathrm{~min}, 4 \mathrm{~min}$ and $5 \mathrm{~min}$ ), and at the same time, carbon dioxide and sodium hydroxide delivery were ceased. Afterwards, stirring was continued for $1 \mathrm{~h}$, and then the mixture was allowed to stand for a certain time. After filtering through quantitative paper, concentrations of remaining calcium ion and magnesium ion in the clear liquor were measured and the amounts of substance were calculated, which were compared to the concentrations and molar amounts of calcium ion and magnesium ion in desalination brine before reaction to calculate the calcium removal rate and magnesium loss rate.

In the second set of experiments, conditions were set identically with the first set of experiments, except that stirring was terminated immediately after completion of reaction. After letting stand for a while, $\mathrm{Ca}^{2+}$ removal rate and $\mathrm{Mg}^{2+}$ loss rate were calculated.

\subsubsection{Preparation of Magnesium Precipitate}

Decalcified mother liquor was added with certain amounts of precipitant sodium hydroxide solution and coagulant aid PAM solution, then reacted, stood still and filtered to give magnesium hydroxide product. By controlling relevant parameters at different levels, the influences of sodium hydroxide addition amount, PAM addition amount, addition order of sodium hydroxide and polyacrylamide and standing time on precipitation effect were investigated.

$100.00 \mathrm{~mL}$ of decalcified mother liquor was accurately pipetted and transferred to a $250 \mathrm{~mL}$ beaker used as the reaction vessel. $p H$ and temperature of the reaction system were adjusted and measured. After certain amounts of sodium hydroxide and PAM solutions were added, $\mathrm{pH}$ and temperature, which stabilized after reaction, were recorded accurately. After letting stand for a while, the mixture was filtered, magnesium ion and calcium ion contents in the filtrate were determined. Optimal proportion was confirmed by continuously changing the molar amount of alkali solution added and the concentration of PAM.

(1) Effect of concentration of PAM solution on magnesium precipitation rate

PAM solutions with mass concentrations of $0.1 \%, 0.2 \%$ and $0.3 \%$ were prepared separately while other pa- 
rameters remained unchanged. Through reaction, precipitation, standing, filtration, titration and calculation, the appropriate concentration of PAM solution was determined.

(2) Effect of addition amount of hydroxide ions on magnesium precipitation rate

Calcium ion and magnesium ion contents in decalcified mother liquor were accurately measured and theoretical molar amount of hydroxide ion required for magnesium ion in $100.00 \mathrm{~mL}$ of decalcified mother liquor was calculated, based on which the molar amount of actually added hydroxide ion was changed continuously. Through reaction, precipitation, standing, filtration, titration and calculation, the appropriate molar amount of hydroxide ion was determined.

(3) Effect of standing time on magnesium precipitation rate

After formation of magnesium hydroxide precipitate, contents of remaining calcium ion and magnesium ion in solution were determined at different standing times, in order to compare the magnesium precipitation rates at different standing times and analyze the effect of standing time on magnesium hydroxide yield.

\subsubsection{Analytical Determination Method}

Titration method was used to determine calcium ion and magnesium ion concentrations [6].

\section{Results and Discussion}

\subsection{Preparation of Decalcified Mother Liquor}

Calcium and magnesium were very similar elements in desalination brine. Carbon dioxide could separate these two elements just because calcium carbonate had a weaker soluability than magnesium carbonate [7]. In this study, we selected the most common temperature $25^{\circ} \mathrm{C}$ as the elementary reacting condition, carbon dioxide was injected as a sufficient reagent to remove calcium from the desalination brine. As can be seen from Table 1, controlling $\mathrm{pH}$ within 7.8 - 8.4 was the primary condition for not less than $90 \%$ yield of calcium carbonate and less than $5 \%$ yield of Magnesium hydrate. If $\mathrm{pH}$ was too low, the resulting calcium carbonate would be easily decomposed; and if $p H$ was too high, calcium carbonate would be easily produced and magnesium loss rate would be high. Compared with $p H$, reaction time was more important to decide magenium loss rate. It was easy to get a calcium removal rate more than $90 \%$, but only reaction time was with $3-4$ minutes could we get a magnesium loss rate less than $5 \%$.

During the carbon dioxide inlet process, calcium carbonate was produced first. As the reaction proceeded, calcium ion concentration in the system decreased gradually. When it decreased to less than $\mathrm{Ksp}_{\mathrm{CaCO}_{3}} /\left[\mathrm{CO}_{3}^{2-}\right]$, carbonate started to react with magnesium ion. So when the reaction time was prolonged to 5 min, the magnesium loss rate increased significantly.

Molecular collision was a key factor in calcium removal. Long duration of molecular collision was a major cause of high calcium removal rate and magnesium loss rate. Magnetic stirring promoted the collision. Stirring time of 3 - 4 min was appropriate to ensure that the high-energy molecules transferred part of the energy to low-energy molecules. Weakest bond received the energy and broke to form effective collision, and thereby produced new substances. When the effective collision was sufficient, the length of precipitate standing time had little effect on the precipitation of calcium and magnesium.

\subsection{Magnesium Extraction by Precipitation Method}

\subsubsection{Effect of PAM Solution Concentration on Magnesium Precipitation Rate}

The amide groups on PAM were interacted by hydrogen bond and then adsorbed the sol particles. After adsorption, PAM chain segments rotated and moved, that is, bridging effect aggregated solid particles together to form precipitate. Therefore, the use of PAM would facilitate the production of magnesium hydrate precipitate.

As can be seen from Figure 1, when the same molar amount of hydroxyl ions was added, the magnesium precipitation rates for the reaction systems added with $0.1 \%$ and $0.2 \%$ PAM were close to that for the reaction system without PAM delivery. This indicated that PAM did not prompt more magnesium in the reaction system to react with hydroxyl anion. In the presence of PAM, the yield did not increase significantly.

In addition, it can also be found from Figure 1 that when the ratio of molar amount of actually added hydroxyl ions to the theoretical value was the same, the magnesium precipitation rate was between $70.48 \%-75.66 \%$ in the presence of 0.3\% PAM, which was less than that in the PAM-free reaction system (greater than $77 \%$ ). When 
Table 1. Calcium removal rate and magnesium loss rate under different conditions.

\begin{tabular}{|c|c|c|c|}
\hline Reaction time/min & $\mathrm{pH}$ & Calcium removal rate/\% & Magnesium loss rate/\% \\
\hline 1 & 7.8 & 90.41 & 0.02 \\
\hline 2 & 7.8 & 91.48 & 1.88 \\
\hline 3 & 7.8 & 97.38 & 2.04 \\
\hline 4 & 7.8 & 98.43 & 2.23 \\
\hline 5 & 7.8 & 97.56 & 2.23 \\
\hline 1 & 8.0 & 91.32 & 0.15 \\
\hline 2 & 8.0 & 94.56 & 1.35 \\
\hline 3 & 8.0 & 97.66 & 3.37 \\
\hline 4 & 8.0 & 98.98 & 3.26 \\
\hline 5 & 8.0 & 98.21 & 4.05 \\
\hline 1 & 8.2 & 93.96 & 0.00 \\
\hline 2 & 8.2 & 96.65 & 0.00 \\
\hline 3 & 8.2 & 99.04 & 1.98 \\
\hline 4 & 8.2 & 99.28 & 4.23 \\
\hline 5 & 8.2 & 99.19 & 60.61 \\
\hline 1 & 8.4 & 94.15 & 0.02 \\
\hline 2 & 8.4 & 97.48 & 2.96 \\
\hline 3 & 8.4 & 99.38 & 75.56 \\
\hline 4 & 8.4 & 99.43 & 77.04 \\
\hline 5 & 8.4 & 99.56 & 78.23 \\
\hline
\end{tabular}

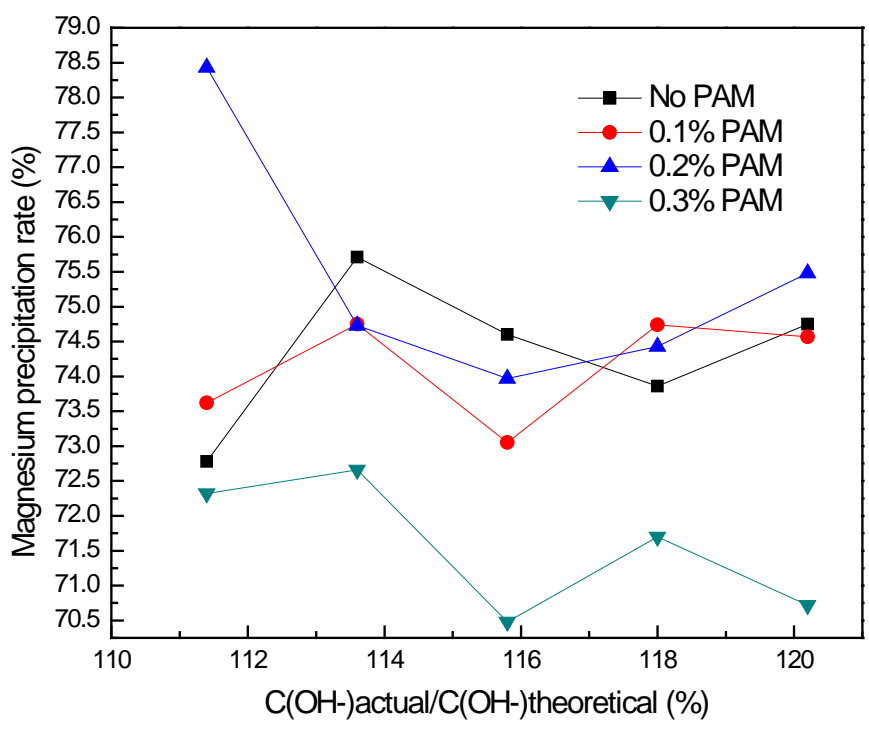

Figure 1. Effect of PAM concentration on magnesium precipitation rate. 
the mass concentration of PAM solution reached $0.3 \%$, the solution had high viscosity, which was mixed slowly and expanded moderately in the reaction system, thus affecting the results. Comprehensive analysis of Figure 1 and Figure 2 showed that the PAM solution well aided the coagulation when its concentration was controlled within $0.1 \%-0.2 \%$.

\subsubsection{Effect of Sodium Hydrate Delivery Amount on Magnsium Precipitation Rate}

As can be seen from Table 2, when the ratio of $\mathrm{n}_{\mathrm{OH}-a c t u a l}$ to $\mathrm{n}_{\mathrm{OH} \text {-theoretical }}$ was $111.8 \%$, magnesium hydrate yield was high. When the ratio of $\mathrm{n}_{\mathrm{OH}-a c t u a l}$ to $\mathrm{n}_{\mathrm{OH} \text {-theoretical }}$ increased to $130 \%$, yield of Magnesium hydrate decreased instead; and at that time, calcium proportion in the precipitate rose.

\subsubsection{Effect of Standing Time on Magnesium Precipitation Rate}

Figure 3 shows changes in magnesium precipitation rate at different standing times. A. Magnesium hydrate yield was $50.25 \%-59.15 \%$ at a standing time of 13 hours; and was $42.74 \%-60.95 \%$ at a standing time of 18 hours. Moreover, the precipitation rate fluctuated widely, indicating insufficient standing and insufficient precipitate production. At a standing time of $60 \mathrm{~h}$, yield was $70.48 \%-77.22 \%$ when the addition amount of hydroxide ion was $101.5 \%-130.2 \%$ of the theoretical value. At a standing time of $65 \mathrm{~h}$, yield ranged from $71.48 \%$ to $74.40 \%$ when the addition amount of hydroxyl anion was $111.4 \%-120.2 \%$ of the theoretical value, which was still slightly lower than that at a standing time of $65 \mathrm{~h}$. At a standing time of $72 \mathrm{~h}$, yield ranged $72.48 \%-75.48 \%$

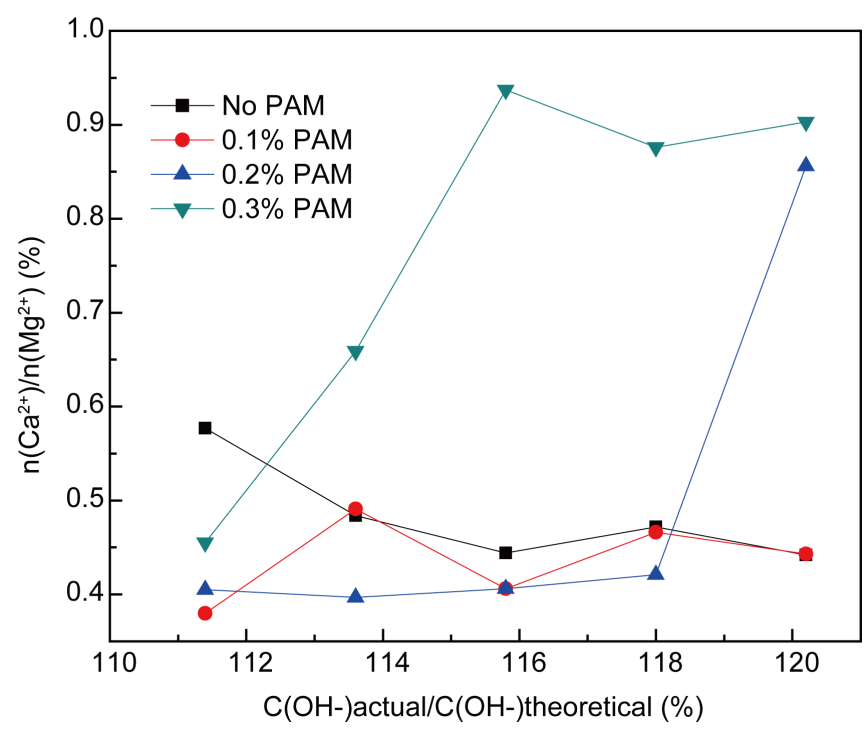

Figure 2. Calcium and magnesium ratio in precipitation over hydroxyl ion dosage.

Table 2. Changes in magnesium precipitation rate with hydroxide ion delivery.

\begin{tabular}{ccc}
\hline$\frac{\mathrm{n}_{\mathrm{OH}-\text { acual }}}{\mathrm{n}_{\mathrm{OH} \text {-theoretical }}} \times 100$ & $\begin{array}{c}\text { Calcium proportion in } \\
\text { precipitation/\% }\end{array}$ & $\begin{array}{c}\text { Magnesium recipitation } \\
\text { rate/\% }\end{array}$ \\
\hline 91.0 & 0 & 52.97 \\
95.1 & 0 & 54.51 \\
101.4 & 0 & 56.31 \\
106.6 & 0 & 58.11 \\
111.8 & 0 & 59.15 \\
130.0 & 1.667 & 50.25 \\
\hline
\end{tabular}




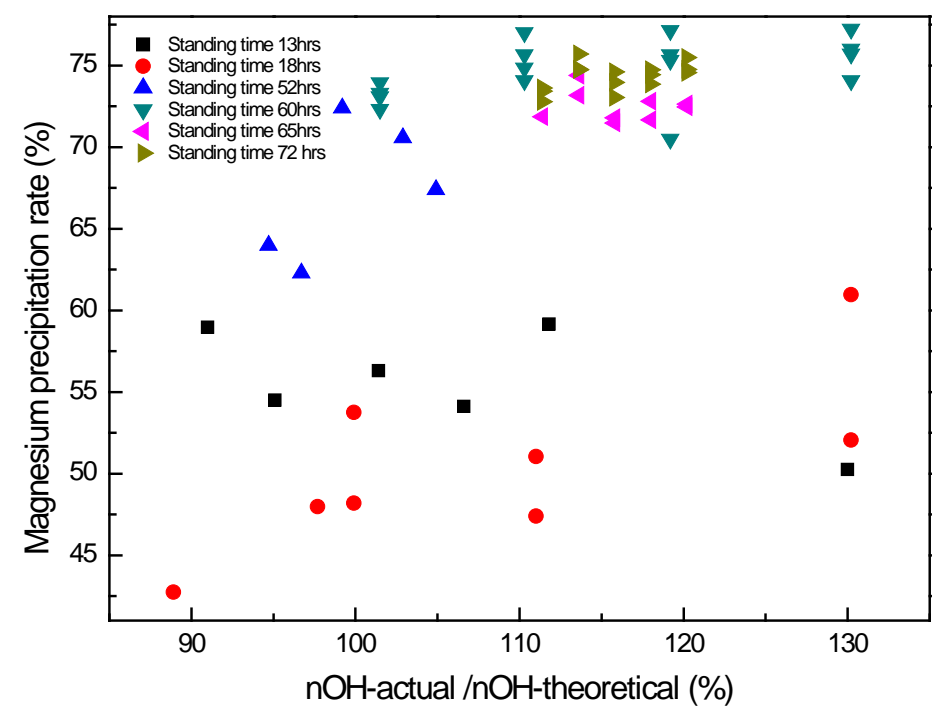

Figure 3. Effect of product standing time on magnesium precipitation rate.

when the addition amount of hydroxide ion was $111.4 \%$ - $120.2 \%$ of the theoretical value. Analysis of precipitation rates at standing times found that extension of standing time contributed little to the increase of precipitation yield at those times.

In addition, calcium ion did not show significant further reduction with increasing molar amount of hydroxide ion addition, indicating that the amount of calcium impurities in the product did not increase significantly. This was because after decalcification treatment, calcium ion concentration was low in the reaction material. Moreover, $\mathrm{Ksp}_{\mathrm{Mg}(\mathrm{OH})_{2}}\left(5.6 \times 10^{-12}, 25^{\circ} \mathrm{C}\right)$ was far smaller than $\mathrm{Ksp}_{\mathrm{Ca}(\mathrm{OH})_{2}}\left(5.5 \times 10^{-6}, 25^{\circ} \mathrm{C}\right)$. Thus, magnesium hydrate precipitate was produced preferentially than calcium hydroxide.

\section{Conclusions}

(1) $0.1 \%-0.2 \%$ PAM solution is added and interacted with the hydrogen bonds in the system to produce bridging effect and promote the precipitation of magnesium hydrate colloid. However, the yield of magnesium hydrate does not improve significantly. When the concentration of PAM solution is low, the molar addition amount of sodium hydroxide solution is a major influencing factor of magnesium hydrate yield. When the ratio of $\mathrm{n}_{\mathrm{OH} \text {-actual }}$ to $\mathrm{n}_{\mathrm{OH} \text {-theoretical }}$ is within a $110 \%-130 \%$ range, magnesium hydrate is largely produced; magnesium precipitation rate is higher than $73 \%$. At the moment alkali solution is added to the decalcified mother liquor, the concentration product of magnesium ion and hydroxyl anion is far greater than $\mathrm{Ksp}_{\mathrm{Mg}(\mathrm{OH})_{2}}$. Magnesium hydrate is produced and hydroxyl anion addition disrupts the kinetic stability of the system, thereby promoting interparticle collisions. Particles lose anti-coagulation stability and coalesce together after collisions, resulting in enlarged particle size, decreased velocity and eventually fomed a kinetically unstable system. Magnesium hydrate particles growed larger and were gradually separated and precipitated from the decalcified mother liquor.

Magnesium in the desalination brine is obtained using sodium hydroxide as the precipitant. When the molar amount of hydroxyl anion is excessive, i.e. between $110 \%-130 \%$, and standing time of precipitate is $60 \mathrm{~h}$, yield of magnesium hydrate exceeds $70 \%$.

Calcium ion in the desalination brine can be effectively removed using carbon dioxide, which thereby improves the purity and reaction efficiency of the magnesium hydrate precipitate. Application of carbon dioxide in extracting magnesium from the desalination brine enables reduction of greenhouse gas emissions while alleviating the environmental problems brought by brine discharge [8] [9].

\section{Acknowledgements}

This study was supported by The National Natural Science Foundation of China (No. 41106066 and No. 41506126). 


\section{References}

[1] Abdul-Wahab, S.A. and Al-Weshahi, M.A. (2009) Brine Management: Substituting Chlorine with On-Site Produced Sodium Hypochlorite for Environmentally Improved Desalination Process. Water Resource Manage, 23, $2437-2454$. http://dx.doi.org/10.1007/s11269-008-9389-7

[2] Chen, X., Chen, X.G., Zhou, Z.F., et al. (2012) Extraction and Modification of Magnesium Hydroxide from Desalination brIne. Guangdong Chemical Industry, 17, 30-31.

[3] Jia, L.L., Chen, H.Y. and Lv, X.L. (2009) Calcium Removal from Concentrated Seawater by Sodium Carbonate in Process of Magnesium Extraction. Inorganic Chemicals Industry, 41, 15-17.

[4] Yu, H. (2011) The Research of $\mathrm{CO}_{2}$ Gas Using in Resource Utilizing of Concentration of Seawater and Softening Seawater. Ocean University of China, Qingdao.

[5] Martinac, V., Labor, M., Mirosevic-Anzulovic, M., et al. (2007) Activated Sinering of Magnesium Oxide Obtained from Seawater. Materials and Technology, 41, 95-98.

[6] Zhu, C.J. (2007) Sawater Analytical Experients. China Ocean University Press, Qingdao.

[7] El-Naas, M.H., Al-Marzouqi, A.H. and Chaalal, O. (2010) Acombined Approach for the Management of Desalination Reject Brine and Capture of $\mathrm{CO}_{2}$. Desalination, 251, 70-74. http://dx.doi.org/10.1016/j.desal.2009.09.141

[8] Zhou, L. and Hu, H.Y. (2013) The Research Progress of the Disposition \& Utilization of Seawater Desalination Brine. International Core Journal of Scientific Research \& Engineering Index, 11, 35-39.

[9] Bond, R. (2010) Zero-Liquid Discharge: Desalination of Waters with High Organic Content. IDA Journal-Desalination and Water Reuse, Second Quarter, 2, 46-52.

\section{Submit or recommend next manuscript to SCIRP and we will provide best service for you:}

Accepting pre-submission inquiries through Email, Facebook, LinkedIn, Twitter, etc. A wide selection of journals (inclusive of 9 subjects, more than 200 journals)

Providing 24-hour high-quality service

User-friendly online submission system

Fair and swift peer-review system

Efficient typesetting and proofreading procedure

Display of the result of downloads and visits, as well as the number of cited articles

Maximum dissemination of your research work

Submit your manuscript at: http://papersubmission.scirp.org/

Or contact gep@scirp.org 